\title{
Délibérations du CC
}

\section{Lors de sa séance d'avril 2009, le Comité central de la FMH a ...}

... approuvé la proposition, destinée à la Chambre médicale, d'introduire la possibilité du postulat pour cette dernière et pour l'Assemblée des délégués;

... nommé M. Gauthey, D. Herren et C. Romann en tant que délégués de la FMH au Sénat de l'Académie suisse des sciences médicales (ASSM);

... approuvé la candidature d'E. Gähler en tant que vice-président de BlueCare SA;

... accepté la mise à disposition du logo FMH pour la Conférence sur le controlling dans le domaine de la santé en Suisse, en Allemagne et en Autriche;

... décidé de donner suite à la demande de parrainage de la Journée de transfert du projet de gestion des risques dans les hôpitaux suisses organisée par l'EPFZ de Zurich et la Haute école de Lucerne;

... accepté d'accueillir en tant qu'hôte l'assemblée annuelle de l'Association Européenne des Médecins des Hôpitaux (AEMH) en 2011;

... pris connaissance de «L'étude sur le besoin d'information des patients en matière de qualité des prestations fournies par les médecins de famille» de la HES bernoise et approuvé la poursuite du projet; il portera sur la question de savoir dans quelle mesure les résultats de l'étude sont représentatifs de l'ensemble de la population;

... pris connaissance du fait que le projet «2010» de Tarmed Suisse ne pourra pas être lancé avant 2010 en raison des mesures de gestion des coûts posées comme préalable par santésuisse;

... décidé que le mandat pour un concept de recherche concomitante sur la tarification SwissDRG sera attribué selon les recommandations du domaine SwissDRG

... approuvé la prise de position relative à la Loi fédérale sur le numéro d'identification des entreprises, car l'attribution d'un numéro IDE pourrait permettre, à l'avenir, de renoncer au numéro de registre des comptes créanciers (RCC);
.. renoncé à prendre position sur la révision de la loi sur l'Assurance-militaire, car les modifications proposées ne touchent pas directement les fournisseurs de prestations;

.. reçu des informations sur les sujets politiques suivants:

- Clause du besoin: l'initiative parlementaire en vue d'une solution transitoire à la clause du besoin proposée par la Commission de la sécurité sociale et de la santé publique du Conseil national a été, dans l'intervalle, acceptée par les deux Chambres. Le sujet est inscrit à l'ordre du jour de la session parlementaire d'été et l'entrée en vigueur est prévue pour janvier 2010. Jusqu'à présent personne n'a appelé à un référendum.

- Managed Care: après avoir clos le chapitre de la clause du besoin, le Parlement s'attelle à une nouvelle tâche visant à fusionner les trois objets liberté de contracter, participation aux coûts et Managed care en une «Réforme de la médecine ambulatoire». Une sous-commission composée de 11 membres, placée sous la direction de la conseillère nationale Ruth Humbel (PDC), est entrée en fonction le 17 avril. La sous-commission entend présenter un texte de modification de la loi dans une année environ.

- Réforme de la loi sur l'assurance-accidents: le projet a été rejeté lors du vote final (en raison de l'obligation de l'affiliation à la SUVA). La majorité de la commission propose de ne pas entrer en matière sur ce point lors de la session parlementaire d'été.

... pris connaissance des développements relatifs aux procédures d'économicité, et approuvé en outre la mise sur pied d'un premier groupe de travail qui se chargera d'élaborer des bases statistiques et un papier de positionnement à l'intention du CC. 